\title{
Robust Learning from Normals for 3D Face Recognition
}

\author{
Ioannis Marras $^{1}$, Stefanos Zafeiriou ${ }^{1, \star}$, and Georgios Tzimiropoulos ${ }^{1,2}$ \\ 1 Department of Computing, Imperial College London, \\ 180 Queen's Gate. London SW7 2AZ, U.K. \\ 2 School of Computer Science, University of Lincoln, \\ Lincoln LN6 7TS, U.K. \\ \{i.marras,s.zafeiriou,gt204\}@imperial.ac.uk \\ http://ibug.doc.ic.ac.uk
}

\begin{abstract}
We introduce novel subspace-based methods for learning from the azimuth angle of surface normals for 3D face recognition. We show that the normal azimuth angles combined with Principal Component Analysis (PCA) using a cosine-based distance measure can be used for robust face recognition from facial surfaces. The proposed algorithms are well-suited for all types of 3D facial data including data produced by range cameras (depth images), photometric stereo (PS) and shade-from-X (SfX) algorithms. We demonstrate the robustness of the proposed algorithms both in 3D face reconstruction from synthetically occluded samples, as well as, in face recognition using the FRGC v2 3D face database and the recently collected Photoface database where the proposed method achieves state-of-the-art results. An important aspect of our method is that it can achieve good face recognition/verification performance by using raw 3D scans without any heavy preprocessing (i.e., model fitting, surface smoothing etc.).
\end{abstract}

\section{Introduction}

3D face recognition from 3D surface information has witnessed very rapid development over the past decade due to the low cost of 3D digital acquisition devices and the need of secure biometrics. Facial surface information has the obvious advantage that it is an intrinsic property of the face and hence more robust to illumination changes than appearance information. On the other hand, 3D face recognition algorithms must be able to handle many challenges such as inaccurate face alignment, pose variations, measurement noise, missing data, facial expressions and partial occlusion.

Many different approaches were proposed for dealing with the aforementioned problems [4 26 6 7 [5 12]. Early approaches, such as [6], use specific face regions that are not affected by the presence of facial deformations caused by facial expressions, such as the nose and the area around it. An investigation on which face regions should be used for robust 3D face recognition was conducted in [7].

Many methods assume that facial expressions can be modeled, up to a certain extend, as isometries of the facial surfaces [2|16|5]. That is, geodesics of the facial surface are

\footnotetext{
* The work of Stefanos Zafeiriou has been partially funded by the Junior Research Fellowship of Imperial College London.
} 
approximately preserved in expressive faces. Following this line of research, the algorithms [216] compare 3D facial surfaces by computing of iso-geodesic stripes or geodesic lengths between closed curves on the facial surface. Under the same assumption, 3D face recognition methods that embed the facial surface into a Euclidean space and replace the geodesic distances by Euclidean ones were proposed in [5]. Using such an embedding, facial surfaces can be matched using conventional methods. The major drawback of these methods is the high computational complexity related to the computation of geodesics. Another solution is to an annotated morphable model [12 143]. One of the drawbacks of these methods is that performance depends on the success of the fitting algorithm which often fails in cases of large expressions or occlusions.

Subspace learning algorithms for normals, such as Principal Component Analysis (PCA), employ low-dimensional representation of surfaces [9 11/17/18]. In its simplest form, PCA on surface normals has been applied on the concatenation of normal coordinates [11]. One attempt to exploit the special structure of normals (i.e., that lie on a sphere) was conducted in [17]. In this paper the Azimuthal Equidistance Projection (AEP) was proposed and applied to surface normals prior to the application of PCA. Furthermore, in order to take into consideration the non-Euclidean actual manifold of objects' surfaces, Principal Geodesic Analysis (PGA) has been proposed [9] for nonlinear statistical analysis.

In this paper, motivated by the recent developments in robust 2D subspace learning from image gradient orientations [19], we propose a simple, efficient and robust subspace learning based on the azimuth angle of face surface normals. We develop a PCA in a space where the normal azimuth angles are mapped by a complex transform into a high-dimensional unit sphere and we demonstrate its robust properties.

The major advantages of the proposed methodology are:

- low-computational complexity. Our algorithms require the eigen-decomposition of Hermitian matrices for training and use simple projections for testing;

- good recognition rates without heavy preprocessing of the 3D meshes (without fitting a model). Comparable performance to state-of-the-art was also achieved when combined with this kind of preprocessing;

- state-of-the-art recognition rates in surfaces produced by Shape-from-X and Photometric Stereo algorithms;

- state-of-the-art recognition rates for cases when standard approaches, such as model fitting, may fail (i.e., presence of occlusions).

\section{PCA of Needle Maps}

Let us define a real surface $f(\mathbf{x})$ on a lattice or a real space $\mathbf{x}=(x, y)$. The normal field (or needle map) is defined as a set of local surface normals $\mathbf{n}(\mathbf{x})=\left(n_{x}(\mathbf{x})\right.$, $\left.n_{y}(\mathbf{x}), n_{z}(\mathbf{x})\right)$ that lie on the unit sphere (i.e., $\|\mathbf{n}(\mathbf{x})\|=1$, where $\|$.$\| is the \ell_{2}$ norm). Mathematically, given a continuous surface $f(\mathbf{x})$, normals $\mathbf{n}(\mathbf{x})$ can be defined as $\mathbf{n}(\mathbf{x})=\frac{1}{\sqrt{1+\frac{\partial f}{\partial x}^{2}+\frac{\partial f}{\partial y}^{2}}}\left(-\frac{\partial f}{\partial x},-\frac{\partial f}{\partial y}, 1\right)^{T}$.

Normals $\mathbf{n} \in \Re^{3}$ do not lie on a Euclidean space but on a spherical manifold $\boldsymbol{\eta} \in \mathcal{S}^{2}$, where $\mathcal{S}^{2}$ is the unit 2-sphere and there is a continuous map $\Phi$ such that $\mathbf{n}=\Phi(\boldsymbol{\eta})$ where 
$\Phi: \mathcal{S}^{2} \mapsto \Re^{3}$. On the unit sphere, the surface normal $\mathbf{n}(\mathbf{x})$ at $\mathbf{x}$ has elevation angles $\theta(\mathbf{x})=\frac{\pi}{2}-\arcsin \left(n_{z}(\mathbf{x})\right)$ and azimuth angle defined as $\phi(\mathbf{x})=\arctan \frac{n_{y}(\mathbf{x})}{n_{x}(\mathbf{x})}=$ $\arctan \frac{\frac{\partial f}{\partial y}}{\frac{\partial f}{\partial x}}$.

Methods for computing the normals of surfaces can be found in [10]. In many cases, surface reconstruction methods do not recover the actual surface but the needle map. Such methods include SfX and PS algorithms [1].

Prior to describing our algorithm, we will briefly outline two popular PCA methodologies on surface normals that take into account the special structure of surface normals (i.e., that lie in a unit sphere). The first one is based on Azimuthal Equidistant Projection (AEP) [17] and the second one on Principal Geodesic Analysis (PGA) [9 18].

For simplicity, hereinafter, we assume that we have a set $\mathcal{J}=\left\{\mathbf{J}_{1}, \ldots, \mathbf{J}_{N}\right\}$ of 3D (or 2.5D) of facial surfaces from a range camera sampled over a grid of resolution $M_{1} \times M_{2}$. For the $i$-th facial surface, at each point of the grid $\mathbf{x}$, we compute the normal vector $\mathbf{G}_{i}=\left[\mathbf{n}_{i}(\mathbf{x})\right] \in \mathcal{P}^{M_{1} \times M_{2}}$ where $\mathcal{P}$ is the pure subset of $\Re^{3}$ of the vector that lies on the unit sphere. In PS and SfX algorithms the set $\mathcal{G}=\left\{\mathbf{G}_{1}, \ldots, \mathbf{G}_{N}\right\}$ is computed directly.

\subsection{PCA Using AEP}

In order to formulate AEP we first need to define the mean elevation and azimuthal angle of $\mathcal{G}$ at each spatial location $\mathrm{x}$. In [17], the mean elevation and azimuthal angles at $\mathbf{x}$ were defined as $\tilde{\theta}(\mathbf{x})=\frac{\pi}{2}-\arcsin \left(\tilde{n}_{z}(\mathbf{x})\right)$ and $\tilde{\phi}(\mathbf{x})=\arctan \frac{\tilde{n}_{y}(\mathbf{x})}{\tilde{n}_{x}(\mathbf{x})}$, where $\tilde{\mathbf{n}}(\mathbf{x})=$ $\left(\tilde{n}_{x}(\mathbf{x}), \tilde{n}_{y}(\mathbf{x}), \tilde{n}_{z}(\mathbf{x})\right)$ is the mean representation of the normal at $\mathbf{x}$. For spherical data, such as surface normals, the intrinsic mean is in many cases represented by the spherical median [8]. For computation ease, in [17], instead of the spherical median, the average surface normal at $\mathbf{x} \mathbf{n}(\mathbf{x})=\frac{1}{K} \sum_{i=1}^{K} \mathbf{n}_{i}(\mathbf{x}), \tilde{\mathbf{n}}=\frac{\dot{\mathbf{n}}(\mathbf{x})}{\|\dot{\mathbf{n}}(\mathbf{x})\|}$ was used.

In order to build the AEP, the tangent plane to the unit-sphere at the location corresponding to the mean-surface normal is computed. A local coordinate system is then established on this tangent plane. The origin is the point of contact between the tangent plane and the unit sphere. The $x$-axis is aligned parallel to the local circle of latitude on the unit-sphere. The AEP maps the normal $\mathbf{n}_{i}(\mathbf{x})$ at $\mathbf{x}$ to the new vector $\mathbf{v}_{i}(\mathbf{x})=\left(v_{x}^{i}(\mathbf{x}), v_{y}^{i}(\mathbf{x})\right)$ (for more details on how to compute the AEP the interested reader may refer to [17]).

After applying the AEP transform to all the samples of $\mathcal{G}$, the matrix $\mathbf{U}=\left[\mathbf{u}_{1} \ldots \mathbf{u}_{N}\right]$ where $\mathbf{u}_{i}=\left[v_{x}^{i}([1,1]) \ldots v_{x}^{i}\left(\left[M_{1}, M_{2}\right]\right) v_{y}^{i}([1,1]) \ldots v_{y}^{i}\left(\left[M_{1}, M_{2}\right]\right)\right]^{T} \in \Re^{M_{1} M_{2}}$ is formed. Finally, the method [17] proceeds by computing the eigenvectors of the covariance matrix $\boldsymbol{\Sigma}_{A E P}=\frac{1}{N} \mathbf{U} \mathbf{U}^{T}$. These eigenvectors $\mathbf{P}$ are used in order to represent facial shape and used as a prior for SfX algorithms. In order to project a novel sample to the subspace of $\mathbf{P}$, we first transform the test normal field $\mathcal{G}$ and $\tilde{\mathbf{n}}(\mathbf{x})$ into $\mathbf{u}$ and then project using $\mathbf{b}=\mathbf{P}^{T} \mathbf{u}$.

\subsection{Principal Geodesic Analysis}

PGA is another statistical analysis method suitable for data that do not naturally lie in a Euclidean space. In standard PCA, the lower-dimensional subspaces form a linear 
subspace in which the data lies. In PGA, this notion is replaced by a geodesic submanifold. In other words, while each principal axis in PCA is a straight line, in PGA each principal axis is a geodesic curve. In the spherical case this corresponds to a circle. PGA utilizes the so-called log and exponential transforms in order to map the normals, that originally lie in a unit sphere, to a space where computing linear variations from the eigenanalysis of a covariance matrix could be meaningful.

In order to formulate PGA first we need to define the exponential and log maps on the sphere and a mean representation of the normals at $\mathrm{x}$. Let $\nu \in T_{n} \mathcal{S}^{2}$ be a vector on the tangent plane to $\mathcal{S}^{2}$ at $\boldsymbol{\eta} \in \mathcal{S}^{2}$ and $\boldsymbol{\nu} \neq 0$. The exponential map of $\boldsymbol{\nu}$, denoted by $\operatorname{Exp}_{\boldsymbol{\eta}}(\boldsymbol{\nu})$, is the point on $\mathcal{S}^{2}$ along the geodesic in the direction of $\boldsymbol{\nu}$ at distance $\|\boldsymbol{\nu}\|$ from $\boldsymbol{\eta}$. The log map is the inverse transform of the exponential map, that is $\log _{\boldsymbol{\eta}}\left(\operatorname{Exp}_{\boldsymbol{\eta}}(\boldsymbol{\nu})\right)=\boldsymbol{\nu}$. The geodesic distance between two points $\boldsymbol{\eta}_{1} \in \mathcal{S}^{2}$ and $\boldsymbol{\eta}_{2} \in \mathcal{S}^{2}$ can be expressed in terms of the log map, i.e. $d\left(\boldsymbol{\eta}_{1}, \boldsymbol{\eta}_{2}\right)=\left\|\log \boldsymbol{\eta}_{1}\left(\boldsymbol{\eta}_{2}\right)\right\|$.

Instead of computing the mean of spherical directional data in [9]18], PGA finds the intrinsic mean, or the so-called spherical median $\boldsymbol{\mu}=[\boldsymbol{\mu}(\mathbf{x})]$ using the Exp and Log mappings. The point $\boldsymbol{\mu}$ cannot be found analytically and a gradient descent procedure was used. For details on the computation of the intrinsic mean and the spherical median, the interested reader may refer to [9].

Having computed the spherical median $\boldsymbol{\mu}$ it was shown that principal geodesics can be approximated by applying linear PCA on the vectors $\mathbf{u}_{\boldsymbol{\mu}}=\left[\boldsymbol{\nu} \boldsymbol{\mu}(1,1)^{T} \ldots\right.$ $\left.\boldsymbol{\nu} \boldsymbol{\mu}\left(M_{1}, M_{2}\right)^{T}\right]^{T} \in \Re^{2 M_{1} M_{2}}$ where $\boldsymbol{\nu} \boldsymbol{\mu}(\mathbf{x})=\log _{\boldsymbol{\mu}(\mathbf{x})}\left(\mathbf{n}_{i}(\mathbf{x})\right) \in \Re^{2}$. After the transformation of the training set $\mathcal{G}$ into the matrix $\mathbf{U}=\left[\mathbf{u}_{\boldsymbol{\mu}}^{1} \ldots \mathbf{u}_{\boldsymbol{\mu}}^{N}\right]$ and then we compute the principal components of $\boldsymbol{\Sigma}_{P G A}=\frac{1}{N} \mathbf{U} \mathbf{U}^{T}$. A normal field of a novel sample $\mathbf{G}$ is transformed using $\log _{\boldsymbol{\mu}}$ into $\mathbf{u} \boldsymbol{\mu}$ and then projected into the subspace $\mathbf{b}=\mathbf{P}^{T} \mathbf{u} \boldsymbol{\mu}$.

\subsection{AAPCA: PCA of Azimuth Angles of Normals}

The proposed PCA of azimuth angles of normals, which we call Azimuth Angle Principal Component Analysis (AAPCA) has been inspired by the use of a complex PCA of image gradient orientations for performing $2 \mathrm{D}$ face recognition robust to occlusions and illuminations [19]. In [19] the distribution of differences of gradient orientations between dissimilar images has been studied and it was shown, with extensive experiments, that this distribution approximately matches a uniform distribution in $[0,2 \pi)$. It was empirically shown that local orientation mismatches caused by outliers can be also well-described by a uniform distribution which, under some mild assumptions, is canceled out by applying the the cosine kernel. The use of cosine kernel then inspired a complex circular PCA. In the following, we show how this concept can be applied for the case of the azimuth angles of normals. To our knowledge, this is the first time that these concepts are introduced for 3D face recognition.

Let us first define the cosine-based dissimilarity measure between two vectors of azimuth angles $\phi_{i}=\left[\phi_{i}(1,1) \ldots \phi_{i}\left(M_{1}, M 2\right)\right]^{T}$ and $\phi_{j}=\left[\phi_{j}(1,1) \ldots \phi_{j}\left(M_{1}, M 2\right)\right]^{T}$ as:

$$
d^{2}\left(\boldsymbol{\phi}_{i}, \boldsymbol{\phi}_{j}\right) \triangleq \sum_{\mathbf{x}}\left\{1-\cos \left[\phi_{i}(\mathbf{x})-\phi_{j}(\mathbf{x})\right]\right\}=\frac{1}{2}\left\|e^{j \boldsymbol{\phi}_{i}}-e^{j \boldsymbol{\phi}_{j}}\right\|^{2}
$$


where $e^{j \phi_{i}}=\left[e^{j \phi_{i}(1)}, \ldots, e^{j \phi_{i}(p)}\right]^{T}$ where $e^{j a}=\cos a+\sqrt{-1} \sin a$. We define the mapping from $[0,2 \pi)^{M_{1} M_{2}}$ to a subset of complex sphere with radius $\sqrt{M_{1} M_{2}}$

$$
\mathbf{z}_{i}\left(\phi_{i}\right)=e^{j \phi_{i}}
$$

After transforming the data we apply PCA on $\mathbf{z}_{i}$ [19], which we call it Azimuth Angle Principal Component Analysis (AAPCA).

\section{Demonstrating the Robust Properties of AAPCA}

In order to demonstrate the robustness of the proposed AAPCA we have conducted a series of experiments using artificially generated data. More specifically, we used 21 images of one of the subjects of the FRGC v2 database. Along with this set of images, we created a second one containing artificially occluded images. In particular, $20 \%$ of the images ware artificially occluded by a 3D cloth patch placed at random spatial locations (the cloth patch has been taken from one of the images of the FRGC v2 database).

For both the original and the corrupted set, we applied standard $\ell_{2}$ PCA on the depth images, PGA and AEP-PCA on the normals, and the proposed AAPCA. Then, we reconstructed the depth, the normals and the azimuth angles using the first 4 principal components of the employed $\ell_{2}$ PCA, AEP-PCA, PGE, and AAPCA, respectively. Fig. 1 illustrates the quality of reconstruction for one example. The first row of Fig. 1 shows the original images of (from left to right) depth, the first two components of the normals and the azimuth angle. The second row of Fig. 1 shows (from left to right) the corresponding occluded images (by the piece of cloth). The third row of Fig. 1 shows the reconstruction of the images in the first row using the 4 principal components of the non-corrupted subspaces. That is, the first image in the third row shows the reconstruction of the non-occluded depth image (the first image in the first row) using the 4 principal components of $\ell_{2}$ PCA. Similarly, the second and third images of the third row show the reconstruction of the first two components of the normals (the second and third images in the first row) using PGA (we show only the PGA result due to space limitation, similar results obtained from AEP-PCA). Finally, the fourth image of the third row shows the reconstruction of azimuth angle using AAPCA. In a similar spirit, the last row of Fig. 11 shows the reconstruction of the occluded set (second row) from the subspaces learned from the corrupted set. For this case, as we may see the reconstruction results, except for the case of the proposed AAPCA, suffer from artifacts.

This result is well justified by looking at the first 4 principal components of each method obtained for both the original and the occluded scenarios. For the latter case, ideally, a robust method would produce eigenvectors that match as closely as possible the ones obtained from the former (original) case. As Fig. 2 shows, this is not the case however for $\ell_{2}$ PCA using depth and PGA. More specifically, in this figure, the first and third row shows the subspace generated by the original images while the second and fourth row shows the subspace generated by the occluded data set. We may observe that in both methods, occlusions result in corrupted subspace. 2] shows also the results of the proposed AAPCA. From the sixth row, it is evident that the principal subspace appears to be artifact-free and therefore dis-occlusion is possible. 
We also evaluated the robust performance of AAPCA quantitatively and compared it with that of PCA on depth values, AEP, and PGA. Because these methods operate on different domains, we used a performance measure which does not depend on the specific domain. More specifically, for each of these methods, we computed a measure of total similarity between the principal subspace for the noise-free case $\mathbf{U}_{\text {noise-free }}$ and the principal subspace for the noisy case $\mathbf{U}_{\text {noisy }}$ as follows:

$$
Q=\sum_{i=1}^{k} \sum_{j=1}^{k} \cos \alpha_{i j},
$$

where $\alpha_{i j}$ is the angle between each of the $k$ eigenvectors defining the principal components of $\mathbf{U}_{\text {noise-free }}$ and each one of $\mathbf{U}_{\text {noisy }}$ [13]. The value $Q$ lies between $k$ (coincident spaces) and 0 (orthogonal spaces) [13]. The mean $Q$ values over 20 repetitions of the experiment (random placement of the occlusion) and for all tested methods are depicted in Fig. 3 (a). The mean values of $Q$ shows that the proposed AAPCA is far more robust that all the other tested methods.

Finally, we evaluated the recognition performance of our algorithm for the case of increasing synthetic occlusions. We used a set of 100 images of 50 persons from the FRGC v2 database and separated it into two sets. Each set contains one image from all 50 persons. All images of the second set (considered as the test set) were artificially occluded by a cloth patch of increasing size. Fig. 3(b) shows the best recognition rate achieved by all tested methods as a function of the percentage of occlusion. As we may observe, our method features by far the most robust performance with a recognition rate over $80 \%$ even when the percentage of occlusion is about $50 \%$.

\section{Experimental Results}

\subsection{FRGC V2}

We used the FRGC v2 3D face database [15] for 3D face recognition experiments. The database contains 3D face scans acquired using a Minolta 910 laser scanner that produces range images with a resolution of $640 \times 480$ in pixels. Results on the database are often summarized by the verification rate $(\mathrm{VR})$ at the point of receiver operating characteristic (ROC) curve where false accept rate (FAR) is equal to $0.1 \%$. We conducted two experiments. In the first one, we fitted an annotated pre-segmented model in the data [3]. In the second one, we simply aligned the data using the eye coordinates provided by the meta-data of FRGC. The only preprocessing applied was a simple median filter on the depth data. In both types we report the VR at FAR $=0.1 \%$ for the ROC III (the interested reader for more details on the ROC III may refer in [12|20]).

The best results achieved in the ROC III for all the tested methods and the stateof-the-art in 3D face recognition literature are summarized in Table 1 (the results of the other methods are directly taken from the corresponding papers). For the standard protocol test ROC III mask of FRGC v2, we obtained verification rates of around $97.4 \%$ in the first type of experiment where we fitted an annotated model. This result has been achieved by using the proposed manifold learning approach of the azimuth angles. This result is directly comparable to the best published results in ROC III. 

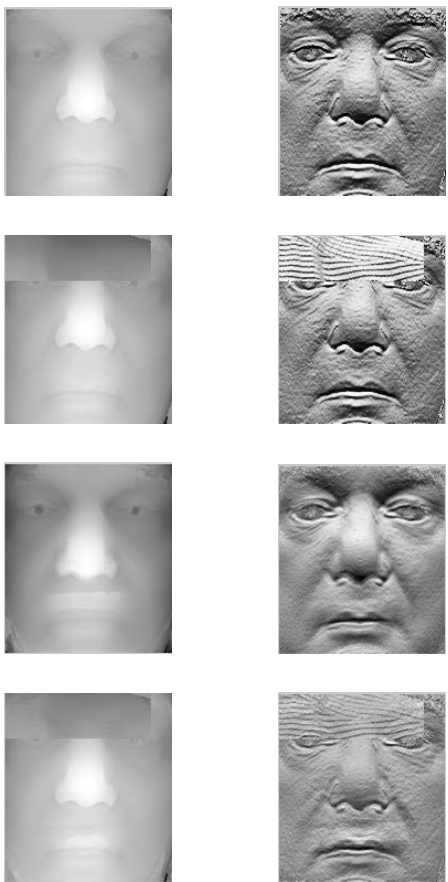
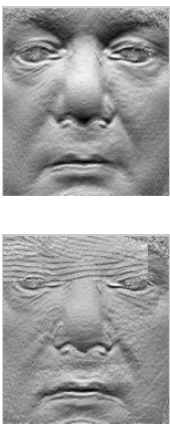
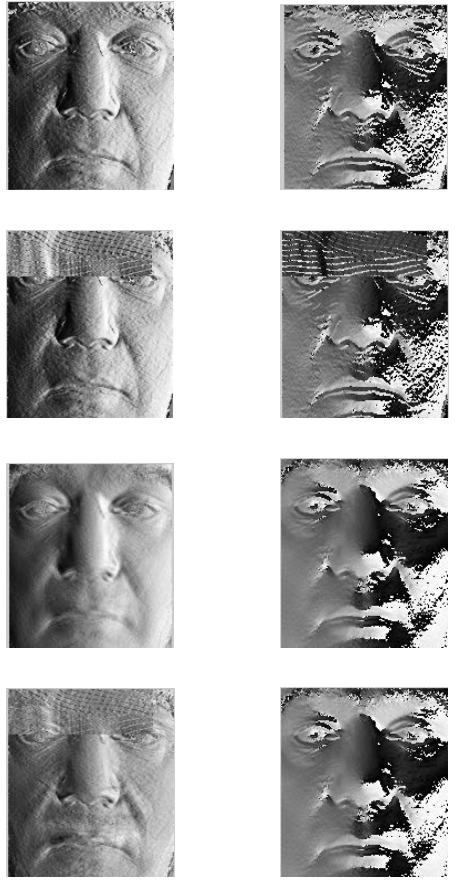

Fig. 1. Quality of reconstruction for $\ell_{2}$ PCA, PGA, and AAPCA. First row: the original images of (from left to right) depth, the first two components of the normals and the azimuth angle. Second row: (from left to right) the corresponding occluded images (by the piece of cloth). Third row: the reconstruction of the images in the first row using the 4 principal components of the non-corrupted subspaces. The first image in the third row shows the reconstruction of the non-occluded depth image (the first image in the first row) using the 4 principal components of $\ell_{2}$ PCA. Similarly, the second and third images of the third row show the reconstruction of the first two components of the normals (the second and third images in the first row) using PGA . Finally, the fourth image of the third row shows the reconstruction of azimuth angle using AAPCA. Fourth row: the reconstruction of the occluded set (second row) from the subspaces learned from the corrupted set. For this case, as we may see the reconstruction results, except for the case of the proposed AAPCA, suffer from artifacts.

In the second experiment, where little preprocessing was applied, the proposed method achieved about $85 \%$ VR at $0.1 \%$ FAR. This VR is quite good if consider that this result can be achieved in less than $0.1 \mathrm{msec}$ per match, while the computational time of fitting a model is on the order of minutes [12/3] in a powerful desktop.

\subsection{Photoface Database}

The Photoface database consists of a total of 1.839 sessions of 261 subjects and a total of 7.356 images. In the verification protocol we applied the probe (or client set) defined by 126 persons. One image is used for training while the second is used for testing 

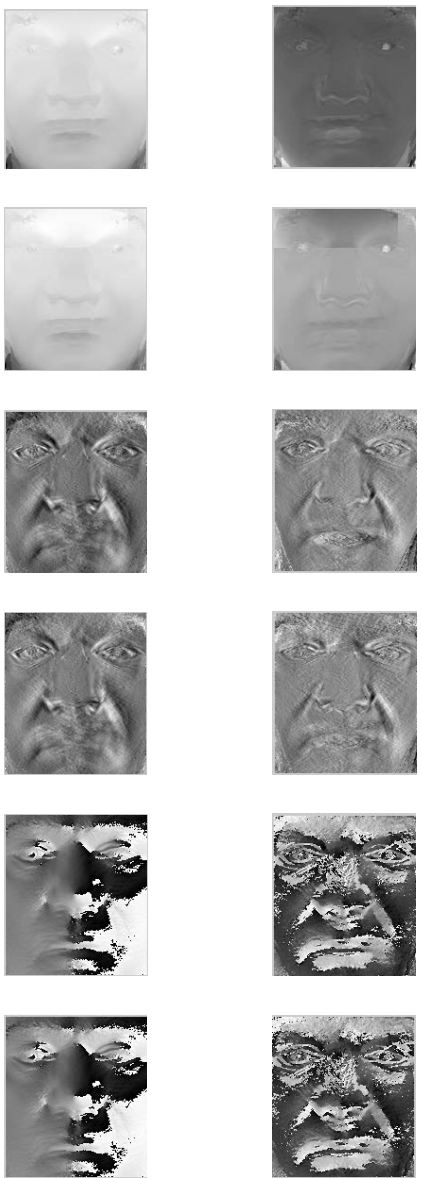
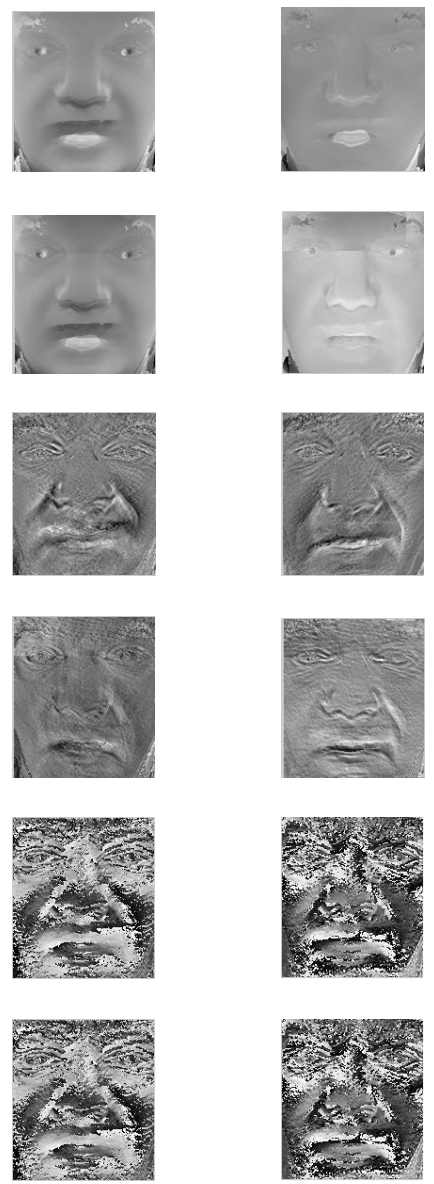

Fig. 2. The 4 principal components of $\ell_{2}$ norm PCA on depth, First row: Original data, Second row: Corrupted data. The 4 principal components of PGA, Third row: Original data, Fourth row: Corrupted data. The 4 principal components of the proposed AAPCA, Fifth row: Original data, Sixth row: Corrupted data.

Table 1. Verification Rate for FAR $=0.1 \%$ in ROC III

\begin{tabular}{|c|c|c|}
\hline & Without Data Pre-processing & With Data Pre-processing \\
\hline Kakadiaris et al. [12] & - & $97.0 \%$ \\
\hline Wang et al. [20] & - & $98.04 \%$ \\
\hline AAPCA & $85.0 \%$ & $97.4 \%$ \\
\hline$\ell_{2}$ PCA & $54.2 \%$ & $91.4 \%$ \\
\hline PGE & $68.3 \%$ & $93.4 \%$ \\
\hline AEP-PCA & $68.3 \%$ & $93.4 \%$ \\
\hline
\end{tabular}



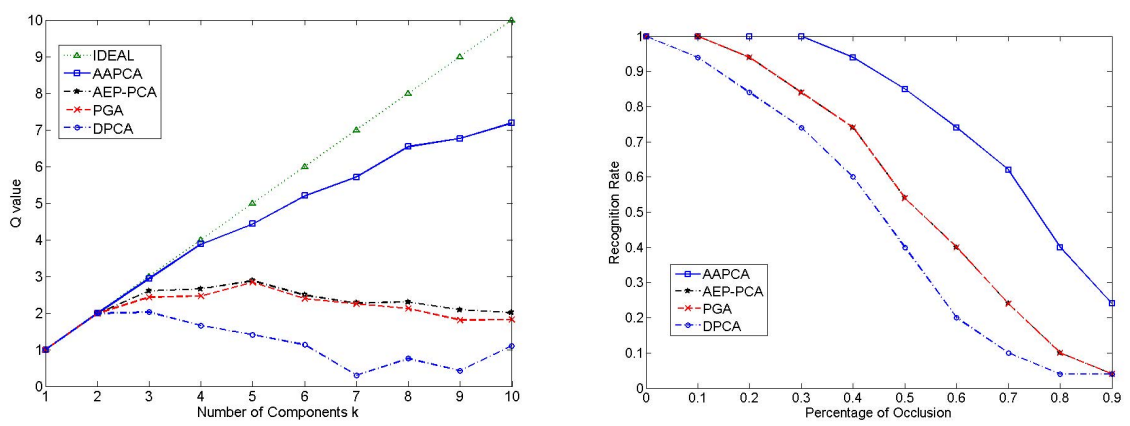

Fig. 3. (a) The $Q$ values obtained for all methods as a function of the number of principal components, (b) Recognition rates for all methods as a function of the percentage of occlusion

client claims. The remaining 135 people in the database, with one image per person, are considered to be impostors. In order to produce the normal field, standard four lights PS was applied with no-preprocessing.

Since, the verification protocol offers only one image for training we applied only PCA-based algorithms. The best Equal Error Rate (EER) for all tested methods are summarized in Table 2 The proposed AAPCA achieved verification performance improvement of more than $40 \%$.

Table 2. EER for the Photoface database

\begin{tabular}{|c|c|c|c|}
\hline Methods & AEP-PCA & PGA & AAPCA \\
\hline EER & $8.3 \%$ & $8.1 \%$ & $4.9 \%$ \\
\hline
\end{tabular}

\section{Conclusions}

We introduced a new concept: PCA of 3D mesh normal orientations. Our framework is as simple as standard $\ell_{2}$ PCA, yet much more powerful for efficient subspace-based data representation. Central to our analysis is the distribution of mesh normal orientation differences and the cosine kernel which provide us a consistent way to measure mesh dissimilarity. We showed how this dissimilarity measure can be naturally used to formulate a robust version of PCA. We demonstrated some of the favorable properties of our framework for the application of 3D face recognition. Extensions of our scheme span a wide range of theoretical topics and applications; from statistical machine learning and clustering to $3 \mathrm{D}$ object recognition.

\section{References}

1. Barsky, S., Petrou, M.: The 4-source photometric stereo technique for three-dimensional surfaces in the presence of highlights and shadows. IEEE T-PAMI 25(10), 1239-1252 (2003)

2. Berretti, S., Del Bimbo, A., Pala, P.: 3D face recognition using isogeodesic stripes. IEEE T-PAMI 32(12), 2162-2177 (2010) 
3. Blanz, V., Scherbaum, K., Seidel, H.: Fitting a morphable model to 3D scans of faces. In: ICCV, pp. 1-8 (2007)

4. Bowyer, K., Chang, K., Flynn, P.: A survey of approaches and challenges in 3D and multimodal 3d+2D face recognition. CVIU 101(1), 1-15 (2006)

5. Bronstein, A., Bronstein, M., Kimmel, R.: Expression-invariant representations of faces. IEEE T-IP 16(1), 188-197 (2007)

6. Chang, K., Bowyer, K., Flynn, P.: Multiple nose region matching for 3D face recognition under varying facial expression. IEEE T-PAMI, 1695-1700 (2006)

7. Faltemier, T., Bowyer, K., Flynn, P.: A region ensemble for 3-D face recognition. IEEE T-IFS 3(1), 62-73 (2008)

8. Fisher, R.: Dispersion on a sphere. Proceedings of the Royal Society of London. Series A. Mathematical and Physical Sciences 217(1130), 295-305 (1953)

9. Fletcher, P., Lu, C., Pizer, S., Joshi, S.: Principal geodesic analysis for the study of nonlinear statistics of shape. IEEE T-MI 23(8), 995-1005 (2004)

10. Foley, J.: Computer graphics: principles and practice. Addison-Wesley Professional (1996)

11. Gokberk, B., Dutagaci, H., Ulas, A., Akarun, L., Sankur, B.: Representation plurality and fusion for 3-D face recognition. IEEE T-SMCS, Part B 38(1), 155-173 (2008)

12. Kakadiaris, I.A., Passalis, G., Toderici, G., Murtuza, N., Lu, Y., Karampatziakis, N., Theoharis, T.: Recognition in the presence of facial expressions: An annotated deformable model approach. IEEE T-PAMI 29(4), 640-649 (2007)

13. Krzanowski, W.: Between-groups comparison of principal components. Journal of the American Statistical Association, 703-707 (1979)

14. Passalis, G., Perakis, P., Theoharis, T., Kakadiaris, I.: Using facial symmetry to handle pose variations in real-world 3D face recognition. IEEE T-PAMI 33(10), 1938-1951 (2011)

15. Phillips, P.J., Flynn, P., Scruggs, T., Bowyer, K.W., Chang, J., Hoffman, K., Marques, J., Min, J., Worek, W.: Overview of the face recognition grand challenge. In: CVPR, pp. 947-954 (2005)

16. Samir, C., Srivastava, A., Daoudi, M.: Three-dimensional face recognition using shapes of facial curves. IEEE T-PAMI 28(11), 1858-1863 (2006)

17. Smith, W., Hancock, E.: Recovering facial shape using a statistical model of surface normal direction. IEEE T-PAMI 28(12), 1914-1930 (2006)

18. Smith, W., Hancock, E.: Facial shape-from-shading and recognition using principal geodesic analysis and robust statistics. IJCV 76(1), 71-91 (2008)

19. Tzimiropoulos, G., Zafeiriou, S., Pantic, M.: Subspace learning from image gradient orientations. IEEE T-PAMI (accepted for publication, 2012)

20. Wang, Y., Liu, J., Tang, X.: Robust 3d face recognition by local shape difference boosting. IEEE T-PAMI 32(10), 1858-1870 (2010) 